\title{
El metoprolol reduce la mortalidad en la insuficiencia cardíaca
}

Effect of metoprolol CR/XL in chronic heart failure: metoprolol CR/XL Randomised Intervention Trial in Congestive Heart Failure (MERIT-HF)

MERIT-HF Study Group. Lancet 1999;353:2001-7.

\section{Objetivo}

Evaluar si el metoprolol de acción prolongada administrado una vez al día y en forma aditiva a la terapéutica habitual, reduce la mortalidad en pacientes con disminución de la fracción de eyección y síntomas de insuficiencia cardíaca.

\section{Diseño}

Estudio clínico controlado multicéntrico, aleatorizado y doble ciego.

\section{Lugar}

Participaron 313 centros pertenecientes a trece países europeos y de Estados Unidos.

\section{Pacientes}

Se incluyeron 3991 pacientes entre 40 y 80 años de edad con diagnóstico de insuficiencia cardíaca clase funcional II - IV, tratados con diuréticos e inhibidores de la enzima convertidora de angiotensina (IECA), estables desde el punto de vista clínico durante las dos semanas previas al ingreso y una fracción de eyección $(\mathrm{FE}) \leq 40 \%$.

\section{Intervención}

Los pacientes fueron asignados en forma aleatoria a recibir metoprolol $(n=1990)$ o placebo $(n=2001)$. La preparación utilizada fue metoprolol $\mathrm{CR} / \mathrm{XL}$, una forma de metoprolol de acción prolongada, y se comenzó con una dosis de 12.5 a $25 \mathrm{mg}$ / día en una o dos tomas diarias. La dosis inicialse aumentaba progresivamente cada dos semanas, según la tolerancia del paciente hasta llegar a una dosis máxima de $200 \mathrm{mg} /$ día.

\section{Medición de resultados principales}

Se consideraron dos resultados principales: mortalidad total y resultado combinado de mortalidad total más internación por cualquier causa. El seguimiento promedio fue de un año y no hubo ninguna pérdida.

\section{Resultados principales}

El estudio fue suspendido precozmente según indicación del comité de seguridad, debido a la observación de una reducción significativa de la mortalidad en el grupo tratado con metoprolol.

La edad promedio fue de 64 años y la dosis promedio de metoprolol utilizada fue de $159 \mathrm{mg} /$ día (64\% de los pacientes recibieron la dosis máxima prefijada de $200 \mathrm{mg} /$ día).

\begin{tabular}{lccccc}
\hline Resultado & $\begin{array}{l}\text { Incidencia } \\
\text { grupo placebo (\%) }\end{array}$ & $\begin{array}{l}\text { Incidencia } \\
\text { grupo Metoprolol (\%) }\end{array}$ & $\begin{array}{c}\text { RRA } \\
(\%)\end{array}$ & $\begin{array}{c}\text { RR } \\
\text { (IC 95\%) }\end{array}$ & $\begin{array}{c}\text { NNT } \\
\text { (IC 95\%) }\end{array}$ \\
\hline $\begin{array}{l}\text { Mortalidad } \\
\text { Total }\end{array}$ & 11 & 7.2 & 3.8 & $\begin{array}{c}0.66 \\
(0.53-0.81)\end{array}$ & $\begin{array}{c}27 \\
(19 \mathrm{a} 48)\end{array}$ \\
\hline $\begin{array}{l}\text { Mortalidad } \\
\text { CV }\end{array}$ & 10 & 6.4 & 3.6 & $\begin{array}{c}0.62 \\
(0.50-0.78)\end{array}$ & $\begin{array}{c}28 \\
(20 \mathrm{a} 46)\end{array}$ \\
\hline
\end{tabular}

Todas las diferencias altamente significativas

La mortalidad total se redujo en un $34 \%$ en el grupo metoprolol con respecto al placebo, mientras que la mortalidad de causa cardiovascular se redujo en un $38 \%$ (ver tabla). La muerte súbita se redujo en el grupo metoprolol en un $41 \%$ ( $4 \%$ vs $6.6 \%$ en el grupo placebo, $p$ 0.0002 , riesgo relativo 0.59 IC $95 \% 0.45-0.78)$. La muerte por progresión de la insuficiencia cardíaca se redujo en un $49 \%$ en el grupo metoprolol ( $1.5 \%$ vs $2.9 \%$ en el grupo placebo, $p$ 0.0023, riesgo relativo 0.51 IC $95 \% 0.33-0.79$ )

La frecuencia de suspensión de la droga fue del $14 \%$ en el grupo metoprolol y $15 \%$ en el grupo placebo.

\section{Conclusiones}

Los autores concluyen que la administración de metoprolol a pacientes estables con IC moderada tratados con IECA y diuréticos reduce la mortalidad total y de causa cardiovascular, tanto por progresión de la IC como por muerte súbita.

\section{COMENTARIO}

El presente estudio demuestra que el tratamiento con metroprolol $\mathrm{CR} / \mathrm{XL}$ de pacientes con insuficiencia cardíaca moderada y disfunción sistólica demostrada, redujo la mortalidad total al año en un $34 \%$ comparado con la administración de placebo. De modo tal que sería necesario tratar con metoprolol a 27 pacientes durante un año para prevenir una muerte.

Esta reducción en la tasa de eventos se observó en forma consistente tanto para mortalidad total, como para mortalidad de causa cardiovascular, muerte súbita y muerte por progresión de la insuficiencia cardíaca.

Cabe hacer notar que la gran mayoría de los pacientes estaban tratados con inhibidores de la ECA por lo que puede considerarse que el efecto beneficioso del betabloqueante es aditivo con respecto a la reducción de la mortalidad ya demostrada con estas drogas.

Si bien el estudio inctuyó pacientes en fase clínica estable, con cla- se funcional II a IV, el $96 \%$ de los mismos se encontraba en clase funcional II-III, por lo que no pueden extraerse conclusiones en relación a pacientes con insuficiencia cardíaca severa.

Los resultados del estudio MERIT son consistentes con los hallazgos del recientemente publicado CIBIS II ${ }^{1}$ que utilizó bisoprolol y con el meta-análisis de Lechat y colaboradores ${ }^{2}$ que incluyó grandes trabajos con betabloqueantes, entre ellos el estudio estadounidense y el estudio australiano con carvedilol. En este momento se puede afirmar que los betabloqueantes tienen una indicación clara en la insuficiencia cardíaca moderada con disfunción sistólica ${ }^{3}$. Su uso requiere un seguimiento estrecho y una lenta progresión de las dosis, ya que aún en menor medida de lo pensado, inicialmente pueden exacerbar la IC en algunos pacientes. Queda por determinar el rol de estas drogas en los pacientes en clase funcional IV y en los pacientes con falla sistólica asintomática.

*Ver Glosario

Dra. Vilma Irazola

Servicio de cardiología. Hospital de Clínicas UBA.

Referencias

1. The cardiac insufficiency bisoprolol study II (CIBIS II). CIBIS II Ivestigators and Committees. Lancet 1999;353:9-13.

2. Clinical effects of beta-adrenergic blockade in chronic heart failure: a meta-analysis of double-blind, placebo-controlled, randomized trials. Lechat P, Packer M, Chalon S et al. Circulation 1998;98(12):1184-91.

3. Beta blocker treatment in heart failure. Carson PE. Prog Cardiovasc Dis 1999;41(4):301-22. 